pre-print, 06. January 2018

\title{
THE DEVELOPMENT OF A HIGH PRECISION TROPOSPHERE EFFECT MITIGATION PROCESSOR FOR SAR INTERFEROMETRY
}

\author{
Nico Adam
}

\author{
German Aerospace Center (DLR), Remote Sensing Technology Institute
}

\begin{abstract}
Troposphere effect mitigation based on numerical weather prediction (NWP) is an actual research topic in SAR interferometry (InSAR) and especially in persistent scatterer interferometry (PSI). This is the reason, a scientific troposphere effect mitigation processing system has been developed. The objective of this paper is to provide the methodology of four developed algorithms, demonstrate application examples, discuss the methods characteristic and recommend techniques for operational systems.
\end{abstract}

Index Terms - persistent scatterer interferometry (PSI), atmospheric phase screen (APS), numerical weather prediction (NWP), wide area processing (WAP)

\section{INTRODUCTION}

Troposphere artefacts are the most dominant error source in InSAR data. In recent years, their mitigation has attracted strong interest starting with [1], [2]. Subject is to improve the measurement precision and therefore provide operationally troposphere corrections with all SAR scenes as proposed by [3] or provide an independent operational service [4]. However, troposphere effect mitigation based on numerical weather prediction (NWP) is still a research topic. This is the reason, an independent scientific troposphere effect mitigation processor (TEMP) has been developed by the author. Four mitigation techniques are implemented and were demonstrated and validated in projects e.g. Terrafirma and a pilot study [5]. Each of these methods has its own characteristic, advantages and limitation. Subject of this paper is to provide the methodology, demonstrate application examples and discuss the methods characteristic.

\section{METHODS}

The Smith-Weintraub equation forms the basis of the NWP based troposphere effect mitigation [6].

$$
N(\vec{r})=k_{1} \cdot \frac{P_{d}(\vec{r})}{T(\vec{r})}+k_{4} \cdot \frac{P_{c}(\vec{r})}{T(\vec{r})}+k_{2} \cdot \frac{e_{w}(\vec{r})}{T(\vec{r})}+k_{3} \cdot \frac{e_{w}(\vec{r})}{T^{2}(\vec{r})}
$$

It models the scaled-up atmospheric refractivity $N(\vec{r})$ by the partial pressures of dry air $P_{d}$ and of carbon-dioxide $P_{c}$, absolute temperature $T$ and the water vapor partial pressure $e_{w}$. Rüger established the scaling constants $k_{l}, k_{2}, k_{3}$ and $k_{4}$ [7]. The range error $d^{N W P}$ can be estimated by the integrated scaled-up refractivity $N(\vec{r})$ along the wave propagation path i.e. the line of sight (LOS)

$$
d^{N W P}=10^{-6} \cdot \int_{\vec{R}_{\text {scatterer }}}^{\vec{R}_{\text {sensor }}} N(\vec{r}) d(\vec{r})
$$

Via the radar wavelength $\lambda$, the atmospheric phase screen (APS) $\varphi^{N W P}$ is calculated by the straight forward relation

$$
\varphi^{N W P}=\frac{4 \pi}{\lambda} d^{N W P}
$$

\subsection{Master selection support}

Using the WRF system [8], the atmosphere state is computed at the time of the SAR acquisition with a resolution of $3 \mathrm{~km} \times 3 \mathrm{~km}$. Now, the scaled-up atmospheric refractivity $N(\vec{r})$ is computed separately for the dry (terms with $P_{d}$ and $P_{c}$ ) and the wet (terms with $e_{w}$ ) component using Eq. (1). For the characterization of the atmosphere, Eq. (2) needs to be implemented. In practice, the zenith direction integration path (Fig. 3) is convenient. A five-point Newton-Cotes integration formula integrates the tabulated data (i.e. the WRF grid, blue points in Fig. 3) fast and without heavy CPU load. A small value of the wet component in the scenes area indicates a suitable master scene. Alternatively, the wavelet transform provides an estimate of the structure function.

\subsection{Vertical stratification mitigation}

At few scene locations, vertical profiles of the troposphere effect are calculated (Fig.1). For this method, the integration along the LOS is required and Eq. (2) is implemented by the Gauss-Kronrod quadrature formula. In practice, the increased computation time of this quadrature algorithm is uncritical because a coarsely sampled grid of vertical profiles (e.g. every $10 \mathrm{~km}$ ) is adequate. Starting from points along the vertical profiles (e.g. every $50 \mathrm{~m}$ ), the troposphere range error is calculated as a function of altitude for the master and slave atmosphere independently. Of course, the height dependent interferometric vertical stratification correction is calculated from the difference of these two values (Fig. 2 left). Typically, a third order polynomial can model this 
function. In the next step, the coefficients of the fitted polynomials are spatially interpolated into the resolution of the input phase resulting in a unique vertical stratification polynomial for each interferogram sample. Similar to DInSAR topography correction, the DEM is transformed into the master scene SAR geometry. Once the actual height at each interferogram sample is available, it is the input argument for the evaluation of the vertical stratification polynomial which provides the correction phase (Fig. 2 right).

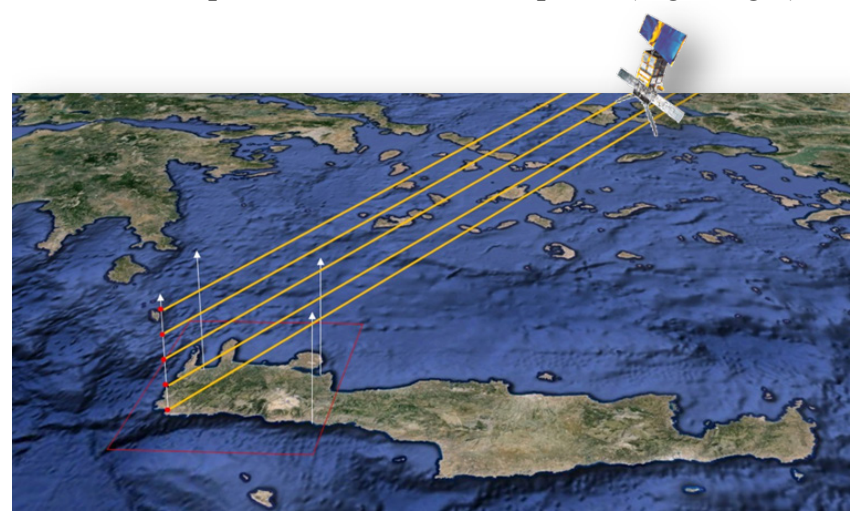

Fig. 1. Principle of vertical stratification mitigation.
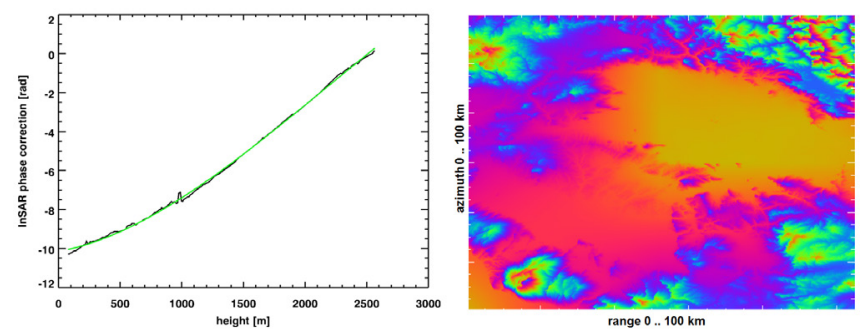

Fig. 2. Interferometric stratification correction. Left: polynomial for a single sample, right: $100 \mathrm{~km}$ x $100 \mathrm{~km}$ interferogram.

\subsection{High resolution APS mitigation}

For high resolution mitigation, the integration along the LOS needs to be performed on the raster of the master scene directly using the local height as the start location. A general problem of a straight forward implementation using the adaptive quadrature is the execution time making it practically infeasible. A newly developed algorithm approximates the integral of each LOS segment (red lines in Fig. 3) between vertical WRF grid layers [9]. The segmentwise parametric integration is based on a linear interpolation of temperature, water vapor and log-linear interpolation of pressure with respect to the orthonormal height and a Taylor series around the center point of the respective interval. As a consequence, the integral for each segment is calculated directly from the intersection values of the LOS with the vertical WRF grid layers (red dots in Fig. 3). An algorithm similar to raytracing provides these intersection points. In doing this, 3D-interpolation and numerous function evaluations can be avoided and the range error $d^{N W P}$ is the sum of few segment integral values.

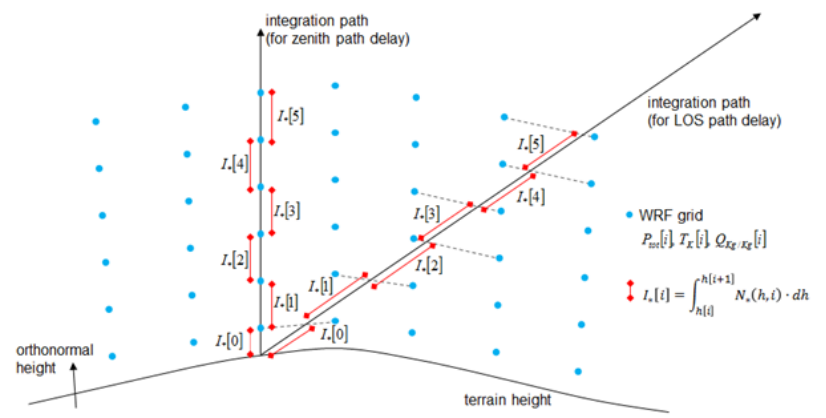

Fig. 3. Integration paths on the NWP data grid.

\subsection{High resolution and high precision APS mitigation}

NWP hindcasts are solutions of partial differential equations and as a result, they are sensitive to initial atmosphere conditions. Similar to chaotic systems, the uncertainty in a hindcast increases exponentially with elapsed time. A typical example is the wind which is imprecise in speed and direction. As a result, humidity and cold or warm air are misplaced at the simulated SAR acquisition time. In order to deal with this effect, ensembles of $N$ hindcasts $\varphi_{i}^{N W P}$ are computed [10]. $\varphi_{i}^{N W P}$ are APS candidates generated from NWP at different times close to the SAR acquisition time. Essentially, a weighted ensemble mean provides the high resolution and high precision APS correction $\hat{\varphi}^{A P S}$

$$
\widehat{\varphi}^{A P S}=\sum_{i=1}^{N}{ }_{i}^{a} \varphi_{i}^{N W P}
$$

The actual implementation estimates additionally to the weights $a_{i}$ a systematic phase component parameterized by $c_{0}, c_{1}$ and $c_{2}$. Effects not part of the NWP can be modelled by this principle e.g. orbit errors and ionospheric effects.

$$
\begin{aligned}
& \underset{\left(c_{*}, a_{i}\right)}{\arg \min } \sum_{i=1}^{N}\left(\varphi^{A P S}-c_{0}-c_{1} \mathbf{x}^{P S}-c_{2} \mathbf{y}^{P S}-a_{i} \varphi_{i}^{N W P}\right) \\
& \text { subject to }: a_{i} \geq 0 \cap \sum_{i}^{N} a_{i}=1
\end{aligned}
$$

${ }^{A P S}$ is the APS estimated from PSI at the locations $\left(\boldsymbol{x}^{P S}, \boldsymbol{y}^{P S}\right)$. Assuming the deformation phase is much smaller in magnitude and extension compared to the APS, the unwrapped D-InSAR phase can be used likewise.

\section{RESULTS}

\subsection{Master selection support}

Fig. 4 compares two geocoded zenith wet effect products taken from ERS acquisitions (white rectangle). The mean values in the scenes areas are $20.7 \mathrm{~mm}$ (left) and $137.9 \mathrm{~mm}$ (right). In this example, the left acquisition taken on Dec 29, 1996 is a better master scene. It follows from the two facts. First, more humidity impacts the radar wave propagation in 
the right acquisition (from Oct 5, 1997). And second, humidity follows a power law which explains the expected higher variation on the high resolution InSAR scale.
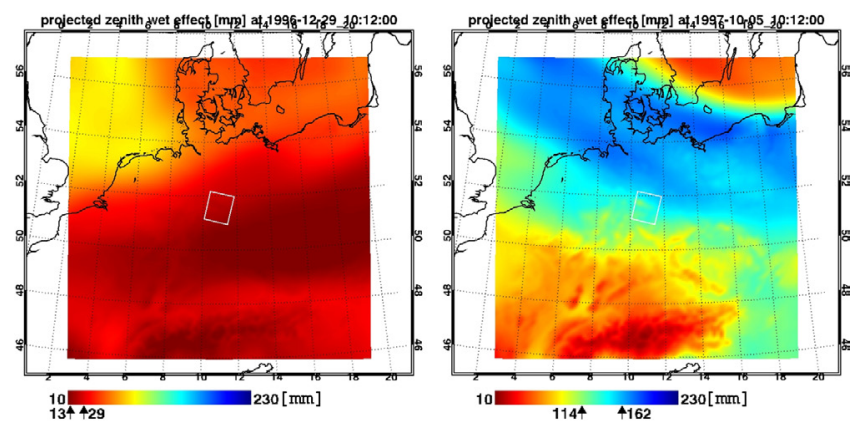

Fig. 4. Geocoded zenith wet effect products.

\subsection{Vertical stratification mitigation}

Fig. 2 (right) visualizes a vertical stratification mitigation phase. It corrects each interferogram sample with an individual value. In plain areas, only little correction is applied. The correction is very smooth in such areas because it uses the NWP hindcast on a very coarse grid only and as a consequence, local atmosphere effects are not mitigated. However, this mitigation is very valuable if the relative estimation spans a height difference. Applying the stratification mitigation, more PSs in mountainous areas can be connected by relative estimates. Fig. 5 provides an example. The periodogram represents the likelihood of an estimate [11] and is visualized for uncorrected (left) and stratification corrected (middle) data. Only in the corrected data (middle), the peak can unambiguously be detected. The coherence improvement depending on the relative height difference has a quadratic characteristic. It is plotted in Fig. 6. For a typical ERS stack, the coherence improves approximately by $10 \%$ for a height difference of $200 \mathrm{~m}$ and by $20 \%$ for $300 \mathrm{~m}$.

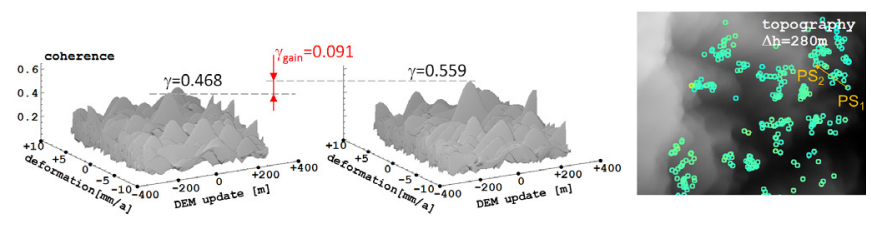

Fig. 5. Periodogram demonstrating an improved estimation.

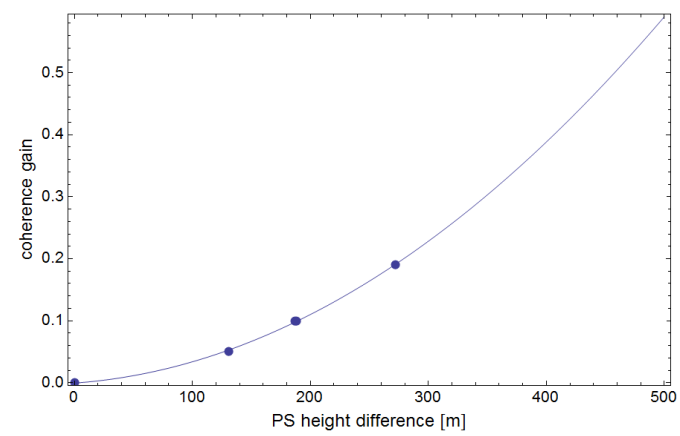

Fig. 6. Coherence improvement depending on height difference.

\subsection{High resolution APS mitigation}

Fig. 9 (bottom left) provides an example for an APS calculated from a NWP hindcast with $3 \mathrm{~km}$ resolution. The test site is in the North German lowlands with very little topography variation. Now, the mitigation phase shows local tropospheric effects. Fig. 7 provides a semivariogram of the residual phase after a PSI processing with uncompensated (solid line) and compensated (dotted line) data. In this example, the SNR of the interferometric data is doubled. It results directly from the high resolution atmosphere correction because of the lack of topography. The semivariogram demonstrates the improvement for a single interferogram. In contrast, the perodogram includes all interferograms and can indicate the improvement for all scenes. Fig. 8 compares the periodograms for the uncompensated (left) and compensated (middle) data. The coherence improves from 0.486 to 0.685 which corresponds to an SNR improvement of about $3.6 \mathrm{~dB}$.

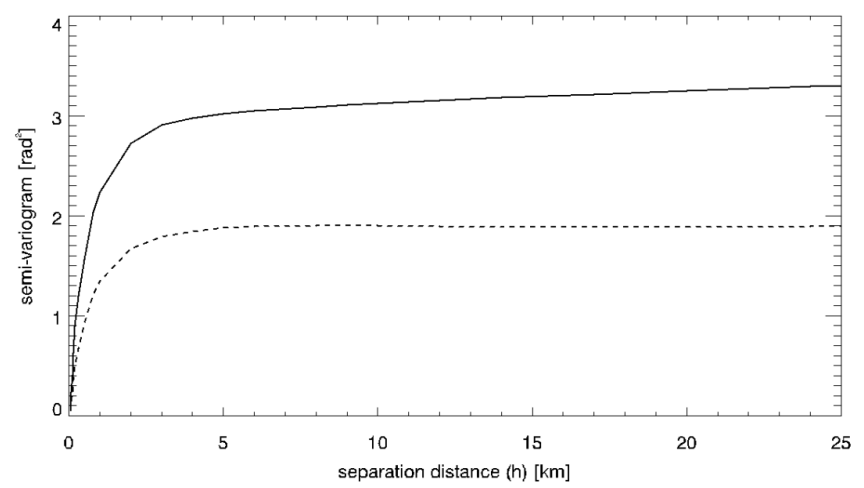

Fig. 7. Semivariogram of uncompensated (solid line) and compensated (dotted line) data.

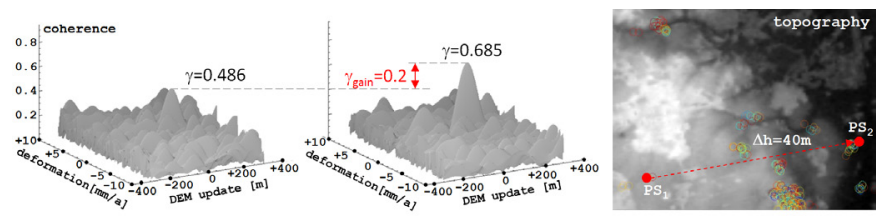

Fig. 8. Periodogram demonstrating an improved estimation.

\subsection{High resolution and high precision APS mitigation}

The weighted ensemble compensates best for the atmospheric effect. The reason is the use of PSI data to correct for the NWP uncertainties. Fig. 9 shows a processing example with the estimated components for an area of 100 $\mathrm{km} \times 100 \mathrm{~km}$. The ensemble of APS candidates is generated by hindcasts with time separations of 15 minutes from each other close to the SAR acquisition time. In this example, the residual phase (i.e. the APS) of the input interferogram spans two phase cycles (upper left picture). However, the compensated interferogram (upper right figure) is within a single phase cycle. 

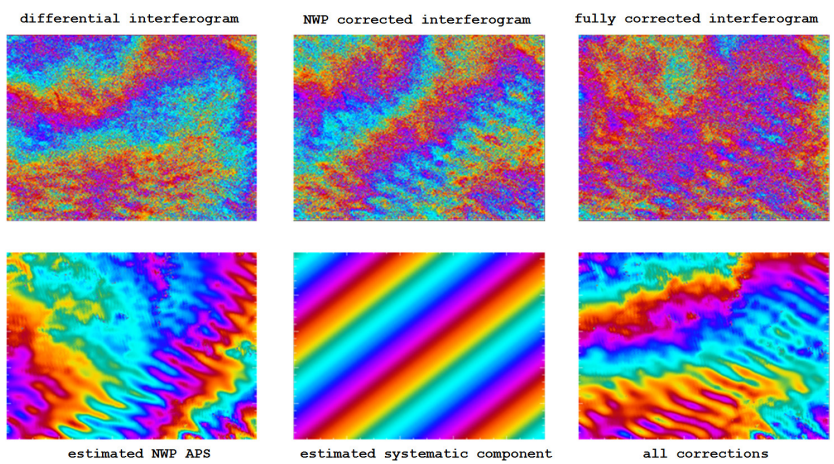

Fig. 9. Estimated components for an area of $100 \mathrm{~km}$ x $100 \mathrm{~km}$.

\section{DISCUSSION}

Four options to implement atmosphere mitigation are presented, namely the master selection support (MSS), the vertical stratification mitigation (VSM), the high resolution APS mitigation (HRM) and the high resolution and high precision APS mitigation (HRPM). All have different characteristics e.g. complexity of implementation, requirements on the hardware and input data, mitigation precision and spatial resolution. In practice, MSS and VSM can be implemented using coarse resolution atmosphere data directly (e.g. CFSR or ECMWF) without the need of a weather model execution. This can reduce the CPU and memory cost compared to the other methods. However, the current implementation in the TEMP framework generates WRF hindcasts with $3 \mathrm{~km}$ resolution because of only moderate hardware requirements. Nowadays, hindcasts with $1 \mathrm{~km}$ resolution are easily available using fast CPUs and CFSR [12] input data. MSS, VSM and HRM are independent of the interferometric data whereas, HRPM utilizes the interferometric phase in order to correct for the NWP uncertainties. As a consequence, the HRPM is available in parallel to a PSI processsing only and not suitable for an independent service. Of course, it provides the best troposphere effect mitigation on a price of being computationally more costly. HRM and HRPM are considered advanced methods due to the mitigation of local troposphere effects with high resolution. At least one of the two should be implemented in an operational system. Practical problems of the high resolution methods are first to require NWP hindcasts from a weather model and second the implementation of the integration Eq. (2) suitable for a dense sampling grid. The newly developed segmentwise parametric integration solves the second problem.

\section{CONCLUSION}

Troposphere effect mitigation is a key methodology in order to improve the InSAR measurement precision, support PSI wide area processing and InSAR applications in mountainous areas. All four presented methods improve the SNR of the interferometric data. For this reason, all techniques are relevant for completed (e.g. ERS-1/2, RADARSAT-1), current (e.g. Sentinel-1, TerraSAR-X) and proposed (e.g. Tandem-L) SAR missions. For operational InSAR systems, it is recommended to implement the MSS and at least one of the advanced methods HRM or HRPM.

\section{REFERENCES}

[1] R. F. Hanssen, "Atmospheric heterogeneities in ERS tandem SAR interferometry", DEOS Report No.98.1, 1998.

[2] R. Holley, G. Wadge, M. Zhu, "New insights into the nature and effects of the water vapour field on InSAR measurements over Etna", FRINGE, accessed 7 Jan 2018, $<$ http://earth.esa.int/fringe07/participants/159/pres 159 holley.pdf $>, 2007$.

[3] M. Eineder, U. Balss, X. Cong, S. Suchandt, C. Gisinger, H. Runge, "Adding Precise Wave-Propagation Information and Geodetic Corrections to Standard SAR Products", CEOS SAR Calibration and Validation Workshop, Noordwijk, Netherlands, 27.-29. Oct. 2015.

[4] P. von Allmen, E. Fielding, E. Fishbein, Z. Li, Z. Xing, L. Pan, "OSCAR: Online Services for Correcting Atmosphere in Radar", accessed 7 Jan 2018. <https://earth.esa.int/ documents/10174/1566700/Allmen FRINGE2011.pdf $\$, 2011$.

[5] A.C. Kalia, M. Frei, T. Lege, "A Copernicus downstream-service for the nationwide monitoring of surface displacements in Germany", In Remote Sensing of Environment, Volume 202, pp. 234-249, 2017.

[6] E. K. Smith and S. Weintraub, "The constants in the equation for atmospheric refractive index at radio frequencies", Proc. IRE, Bd. 41, pp. 1035-1037, 1953.

[7] J. M. Rüeger, "Refractive Index Formulae for RadioWaves", JS28: Integration of Techniques and Corrections to Achieve Accurate Engineering, 19-26 April 2002.

[8] National Center for Atmospheric Research (NCAR), accessed 7 Jan 2018, <https://www.mmm.ucar.edu/weatherresearch-and-forecasting-model>.

[9] N. Adam, ,Algorithmic PSI Improvement in Mountainous Areas by Atmosphere Mitigation", Technical Report. DLR, accessed 7 Jan 2018, <http://elib.dlr.de/ 95190/1/AtmosphereMitigation_v3.10.pdf $>, 2014$.

[10] F. Ulmer and N. Adam, “A Synergy Method to Improve Ensemble Weather Predictions and Differential SAR Interferograms", In ISPRS Journal of Photogrammetry and Remote Sensing, Volume 109, pp. 98-107, 2015.

[11] A. Ferretti, C. Prati, F. Rocca, "Permanent Scatterers in SAR Interferometry", IEEE Transactions on Geoscience and Remote Sensing, vol. 38, pp. 2202 - 2212. 2000.

[12] S. Saha, S. Moorthi, X. Wu, J. Wang, S. Nadiga, P. Tripp, H.-L. Pan, D. Behringer, Y.-T. Hou, H.-y. Chuang, M. Iredell, M. Ek, J. Meng, R. Yang, "The NCEP Climate Forecast System Version 2“, 2011. 